\title{
Interactions between spatial and spatiotemporal information in spatiotemporal boundary formation
}

\author{
DOUGLAS W. CUNNINGHAM \\ Logicon Technical Services, Inc., Dayton, Ohio \\ THOMAS F. SHIPLEY \\ Temple University, Philadelphia, Pennsylvania \\ and \\ PHILIP J. KELLMAN \\ University of California, Los Angeles, California
}

\begin{abstract}
The surface and boundaries of an object generally move in unison, so the motion of a surface could provide information about the motion of its boundaries. Here we report the results of three experiments on spatiotemporal boundary formation that indicate that information about the motion of a surface does influence the formation of its boundaries. In Experiment 1, shape identification at low texture densities was poorer for moving forms in which stationary texture was visible inside than for forms in which the stationary texture was visible only outside. In Experiment 2, the disruption found in Experiment 1 was removed by adding a second external boundary. We hypothesized that the disruption was caused by boundary assignment that perceptually grouped the moving boundary with the static texture. Experiment 3 revealed that accurate information about the motion of the surface facilitated boundary formation only when the motion was seen as coming from the surface of the moving form. Potential mechanisms for surface motion effects in dynamic boundary formation are discussed.
\end{abstract}

The perceptual quality of a scene may be altered substantially by observer or object motion (Gibson, Kaplan, Reynolds, \& Wheeler, 1969; Helmholtz, 1867/1962; Michotte, Thines, \& Crabbé, 1964/1991). For example, in scenes in which surface properties of objects match the background, objects are invisible until they move (Gibson, 1968). They become visible when they move because the changes over time at the edges of an object are sufficient to define those edges (Gibson et al., 1969). Since Gibson and his colleagues demonstrated this effect, other researchers have provided a more detailed analysis of the perception of edges defined by texture transformations like accretion and deletion (Andersen \& Cortese, 1989; Bruno \& Bertamini, 1990; Bruno \& Gerbino, 1991; Cunningham, Shipley, \& Kellman, in press; Hine, 1987; Miyahara \& Cicerone, 1997; Shipley \& Kellman, 1993, 1994, 1997; Stappers, 1989), as well as the perception of surface qualities from element transformations (Cicerone, Hoffman, Gowdy, \& Kim, 1995; Cunningham et al., in press; Miyahara \& Cicerone, 1997) and the perception of depth from accretion and deletion (Kaplan, 1969; Ono, Rogers, Ohmi, \& Ono, 1988; Rogers, 1984; Rogers \&

This research was supported by NSF Research Grants BNS 936309 to T.F.S. and SBR- 9496112 to P.J.K. D.W.C. was at Temple University when this research was conducted. Portions of this research were presented at the 1996 annual meeting of the Association for Research in Vision and Ophthalmology and at the 1994 annual meeting of the American Psychological Society. Correspondence should be addressed to T. F. Shipley, Department of Psychology, Temple University, Philadelphia, PA 19122 (e-mail: tshipley@astro.ocis.temple.edu).
Graham, 1983; Royden, Baker, \& Allman, 1988). Surface perception and edge perception have generally been studied independently. No experiments have been reported that explicitly examine interactions between these two perceptual phenomena. For example, it is unknown whether surface information can influence dynamic edge formation. Several researchers have discussed this issue, but their experiments were not designed to address it directly (Cicerone et al., 1995; Kaplan, 1969; Shipley \& Kellman, 1994). Here, we explore how the information provided by a surface influences dynamic edge perception.

Early work with dynamically defined edges exclusively used the appearance and disappearance of texture elements at the edges of objects. Recent work suggests that accretion and deletion is just one member of a large class of transformations that can define edges (Shipley \& Kellman, 1994). In addition to changes in visibility (i.e., accretion and deletion), changes in color, location, orientation, and shape can all define edges. Shipley and Kellman $(1994,1997)$ suggested that the spatiotemporal pattern of any abrupt change at the edge of a figure can be used to define that edge, a process they refer to as spatiotemporal boundary formation (SBF). The abrupt changes, or spatiotemporal discontinuities, serve as the initiating conditions for SBF. Sequential pairs of spatiotemporal discontinuities define motion signals which can be used to recover the orientation and direction of motion of the edge that caused the change.

Although changes are necessary for SBF, the static texture patterns that arise as the changes occur also appear 
to play some role. Shipley and Kellman (1994) used an objective measure of contour clarity, a shape identification task, to study boundary formation in SBF displays in which the presence of static differences was varied. When static differences between the inside and outside of a surface were present (see Figure 1a), a surface with sharp, well-defined contours and a particular depth location was seen. Such static differences arise when only one type of transformation occurs along a given edge (following Shipley and Kellman, we refer to these displays as "unidirectional"). For example, when white elements on a black background disappeared inside a moving form, a black form was seen sequentially covering and revealing the white elements. In contrast, in bidirectional displays, in which elements were transformed in both directions along any given edge (see Figure $1 \mathrm{c}$ ), no static texture differences were present. In these displays, the contours were less clear and typically did not seem to belong to any surface (Shipley \& Kellman, 1994). Such observations indicate that spatiotemporal discontinuities are sufficient for the perception of boundaries, and static differences may facilitate boundary formation.

The influence of static texture on edge perception in unidirectional and bidirectional displays may be the result of a motion conflict, which arises as a result of differing distributions of stationary texture elements in the two displays. When a real object moves, internal texture generally moves with it. In the bidirectional displays with disappearances and appearances, static elements were visible both inside and outside, so the internal texture did not move with the dynamically defined edge. However, in the unidirectional displays with disappearances and appearances, the static texture was visible only outside the figure, so no conflict was present between the motion of the dynamically defined edge and the motion -or lack thereof - of the internal texture. Accuracy differences between uni- and bidirectional transformations were not seen when transformations of element orientation and location were employed. Such transformations do not result in changes in element visibility (Shipley \& Kellman, 1994). This suggests that changes in visibility of elements may have been the critical factor in Shipley and Kellman's finding. In the present Experiment 1, we investigated the role of visible texture in SBF by systematically varying its location relative to the moving edge.

\section{EXPERIMENT 1}

There are three possible relationships between the location of static texture and a moving edge: Static texture may be visible inside, outside, or on both sides of a moving edge. The first two relationships arise from unidirectional transformations, and the last requires a bidirectional transformation. We refer to these three relationships, all of which were employed in Experiment 1, as texture-inside unidirectional, texture-outside unidirectional, and bidirectional, respectively (see Figure 1). The texture-outside displays replicate the unidirectional displays used by

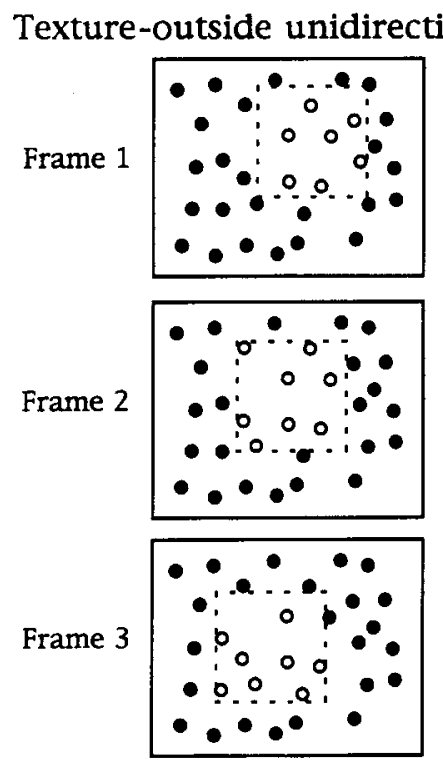

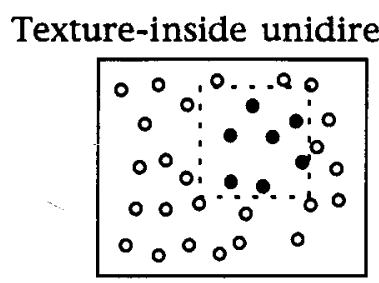
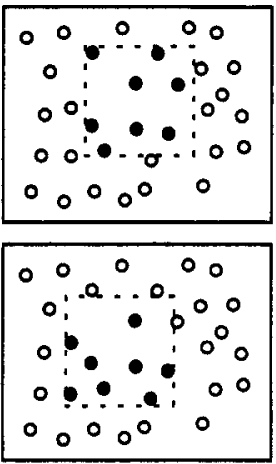

Bidirectional
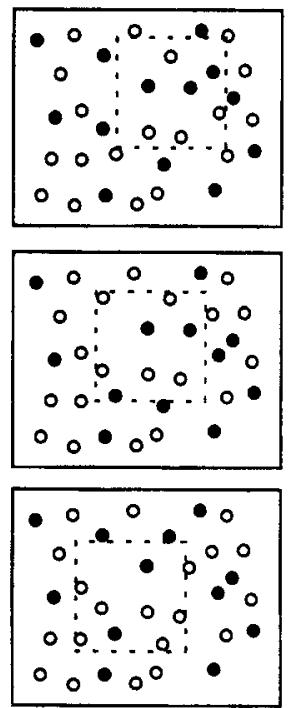

- =Visible texture element

$0=$ Invisible texture element

Figure 1. The three types of transformations used in Experiment 1. (a) In texture-outside displays, the texture elements are progressively hidden and revealed by a moving form. In such displays, texture elements are visible only while outside the moving form. (b) In texture-inside displays, the texture elements are visible inside the moving form. (c) In bidirectional displays, half of the elements are visible only while outside the form. The remaining elements are visible only while inside the moving form. From "Spatiotemporal Boundary Formation: Boundary, Form, and Motion Perception From Transformations of Surface Elements," by T. F. Shipley and P. J. Kellman, 1994, Journal of Experimental Psychology: General, 123, p. 5. Copyright 1994 by the American Psychological Association. Adapted with permission. 
Shipley and Kellman (1994), in which the texture elements were visible only outside the figure. The pattern of transformations in these displays is consistent with the pattern present when a small occluder moves over a speckled background. The texture-inside displays were identical to the texture-outside displays except that the elements were visible only inside the figure. The pattern of transformations in these displays is like the pattern present when a large opaque occluder with a hole in it moves over a speckled background. In the bidirectional displays, texture was visible on both sides of the figure. If the presence of stationary texture inside a moving form influences SBF, then the two unidirectional displays should differ: Accuracy in the texture-outside condition should be superior to that in both the texture-inside and the bidirectional conditions. Since the previously reported difference between texture-outside and bidirectional displays was seen across a broad range of shape identification accuracy, Experiment 1 also duplicated Shipley and Kellman's (1994) density manipulation: The spatial density of texture elements was varied in all three display types.

\section{Method}

Subjects. Eleven Temple University undergraduates participated in partial fulfillment of introductory psychology course requirements. One subject's data were excluded from analysis due to the subject's failure to follow instructions.

Apparatus. All displays were generated and presented by a Macintosh Quadra 800 with an E-machines TX 16 monitor. The monitor was $25 \mathrm{~cm}$ high $\times 33 \mathrm{~cm}$ wide, with a resolution of 34.25 dots per centimeter ( 808 vertical $\times 1,024$ horizontal pixels). The monitor was the sole source of illumination in the room. Subjects were positioned $150 \mathrm{~cm}$ from the monitor.

Stimuli. The displays were sparsely textured random-dot kinematograms. The kinematograms consisted of a $14.6 \times 14.6 \mathrm{~cm}$ (visual angle of $5.58^{\circ}$ ) field of small (diameter $=1.3 \mathrm{~mm}$ 2.98 arc $\mathrm{min}$ ), circular, stationary, white (luminance $=35 \mathrm{~cd} / \mathrm{m}^{2}$ ) elements presented on a black (less than $0.001 \mathrm{~cd} / \mathrm{m}^{2}$ ) background. One of 10 mathematically defined regions (see Figure 2) moved along a circular path (radius $=1.39 \mathrm{arc} \mathrm{deg}$ ) through this field of elements. Whenever the leading edge of the moving form--which we will refer to as a pseudosurface-passed over the center of an element, the entire element was transformed. As long as an element remained within the boundaries of the pseudosurface, the element
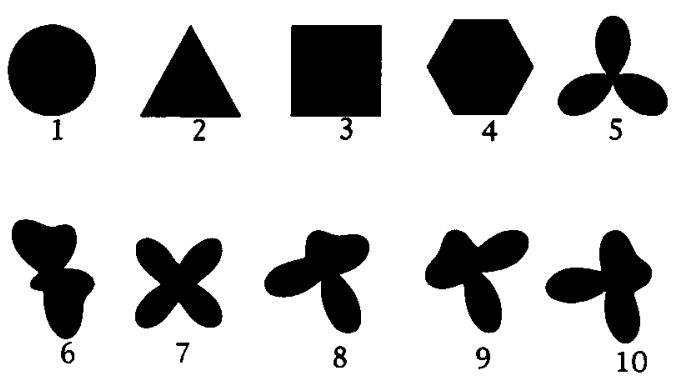

Figure 2. The 10 forms used for a 10-alternative forced-choice shape identification task in Experiment 1. From "Spatiotemporal Boundary Formation: Boundary, Form, and Motion Perception From Transformations of Surface Elements," by T. F. Shipley and P. J. Kellman, 1994, Journal of Experimental Psychology: General, 123, p. 6. Copyright 1994 by the American Psychological Association. Adapted with permission. remained transformed. When the pseudosurface no longer covered the center of an element, the element returned to its original state.

The element field density was systematically varied by distributing 50, 100,200, or 400 elements within the display area; elements covered $0.25 \%, 0.5 \%, 1 \%$, and $2 \%$ of the display area, respectively. Elements were randomly distributed throughout the display area, with the following constraint. In order to prevent large variations in local density, the display area was divided into small, equal-sized, nonoverlapping regions. The elements were distributed randomly within each region, with an equal number of elements in each. When there were 100 or more elements present, 100 regions were used. When 50 elements were present, 49 regions were used.

The pseudosurfaces (subtending on average $2^{\circ}$ ) were designed to provide an objective measure of contour clarity (Figure 2), where greater shape identification reflects perceptually clearer edges. Each pseudosurface was similar in shape to at least one other pseudosurface. All pseudosurfaces were matched for maximum extent, and some were matched for total surface area. Some of the shapes were familiar (e.g., Figure 2, forms 1, 2, and 3) and others were unfamiliar (e.g., Figure 2, forms 8, 9, and 10). Previous research has shown that this set of pseudosurfaces provides a reliable means of identifying variables that influence dynamic edge perception (Shipley \& Kellman, 1993, 1994, 1997).

Three types of displays were employed: texture outside, texture inside, and bidirectional. In the texture-outside displays, all the elements outside of the pseudosurface were white and thus visible, while those inside were black and thus invisible. As the pseudosurface moved, elements appeared at the trailing edges of the form and disappeared at the leading edges (Figure 1a). The texture-inside displays were identical to the texture-outside displays except that the colors of the elements were reversed. Elements were visible only when they were inside the pseudosurface (see Figure $1 \mathrm{~b}$ ). In the bidirectional displays, half of the elements were visible only when they were outside the pseudosurface (as in the texture-outside displays), and the other half were visible only when inside the pseudosurface (as in the texture-inside displays). In these displays, there was no static information about the figure (Figure 1c); at any given instant, the texture density inside the pseudosurface was equivalent to the texture density outside the surface. Although the location of visible elements differed in the three types of displays, the average number of transformations per frame in all three types of displays was identical.

The displays were 60 -frame animation sequences, with each frame displayed for $40 \mathrm{msec}$. To generate each frame, 60 equally spaced positions were chosen along the circular path of the pseudosurface. The positions were $3.8 \mathrm{~mm}(8.7 \mathrm{arc} \mathrm{min})$ apart. For each frame, the position of each element relative to the location of the pseudosurface on that frame (i.e., inside versus outside) was determined, and the element was drawn in the appropriate color.

Each trial consisted of a single animation sequence that was shown continuously for 20 cycles $(48 \mathrm{sec})$ or until the subject responded, whichever came first. Crossing three types of transformation (texture outside, texture inside, and bidirectional), four element field densities $(0.25 \%, 0.5 \%, 1 \%$, and $2 \%)$, and 10 pseudosurfaces yielded 120 trials. The 120 trials were presented in random order.

Procedure. The subjects' task was to identify the shape of the pseudosurface in each display. In this 10 -alternative forced-choice task, subjects selected a static drawing of one of the 10 possible forms (the 10 alternatives were displayed on the left side of the monitor at all times). Subjects were shown an example trial and instructed to identify the correct figure as quickly and accurately as possible. Following that, the 120 trials were presented.

\section{Results}

The presence of stationary texture elements inside a moving form appears to interfere with shape identification. For the displays that did not have texture inside the moving form (texture-outside displays), subjects identified the 
correct figure $80 \%$ of the time (with chance accuracy being $10 \%$ ). In the two types of display that did have texture inside the moving form (texture-inside and bidirectional displays), accuracy was $68 \%$ and $54 \%$, respectively.

Mean accuracies for the three conditions are plotted as a function of element field density in Figure 3. As can be seen in Figure 3, accuracy increased with increases in density, and overall accuracies were lower in the textureinside than in the texture-outside displays, and lower still in the bidirectional displays. A two-way analysis of variance (ANOVA) performed on the accuracy scores, with type of transformation (texture outside, texture inside, or bidirectional) and element field density $(0.25 \%, 0.5 \%$, $1 \%$, or $2 \%$ ) as within-subject factors, confirmed that both main effects were significant $[F(2,18)=42.12, p<$ .0001 , and $F(3,27)=81.43, p<.0001$, respectively].

The superiority of texture-outside over texture-inside displays was most pronounced at the lowest density and disappeared as density increased. This is reflected in a significant transformation $\times$ density interaction $[F(6,54)=$ $4.43, p<.001]$. Planned comparisons (using the error term for the transformation $\times$ density interaction) showed that the accuracy for the texture-inside displays was significantly lower than that for the texture-outside displays at the lowest density $[F(1,54)=35.86, p<.0001]$, but accuracy for the texture-inside displays was not significantly greater than that for the texture-outside displays at the higher three densities $[F(1,54)=3.50, p>.066$ for the $0.5 \%$ density level, and $F<1$ for the $1 \%$ and $2 \%$ density levels]. The opposite pattern was found for the bidirectional and texture-inside displays: Texture-inside accuracy was not significantly different from bidirectional accuracy at the lowest density $(F<1)$, but was signifi- cantly higher at $0.5 \%$ and $1 \%$ (all $F \mathrm{~s}(1,54)>15.40$, all $p s<.0002$ ). The difference between bidirectional and texture-inside accuracy did not reach significance at the highest density $[F(1,54)=2.83, p<.1]$, but this may reflect, at least in part, a ceiling effect. Neither the textureoutside nor the texture-inside accuracy increased significantly from the $1 \%$ to the $2 \%$ density levels (both $F \mathrm{~s}<1$ ), suggesting that subjects may have reached a ceiling in performance on this task.

The texture-outside condition differed from the textureinside and bidirectional conditions in the pattern of shape identification errors. At low densities in the textureinside and bidirectional displays, subjects were more likely to identify a regular smooth form (circle, triangle, and square) as an irregularly shaped form (forms 5-10 in Figure 2) than vice versa [both $\chi^{2} \mathrm{~s}(1, N \geq 81) \geq 4.35, p \mathrm{~s}<$ $.05]$. This was not true in texture-outside displays, where the confusion between smooth and irregular forms was symmetric $\left[\chi^{2}(1, N=88)=1.95, \mathrm{n} . \mathrm{s}\right.$. $]$.

\section{Discussion}

The presence of static texture elements inside a moving form appears to play a role in boundary formation: Contour clarity in both the bidirectional displays and the texture-inside displays was inferior to contour clarity in the texture-outside displays. Additionally, an instability in boundary formation was apparent in the phenomenal appearance of the texture-inside displays. The contours in these displays appeared to fluctuate nonrigidly, in an amoeba-like manner, in the lower densities. In contrast, the contours in the texture-outside displays appeared to be stable and relatively clear, even at the lower densities. Additionally, the differences in boundary formation be-

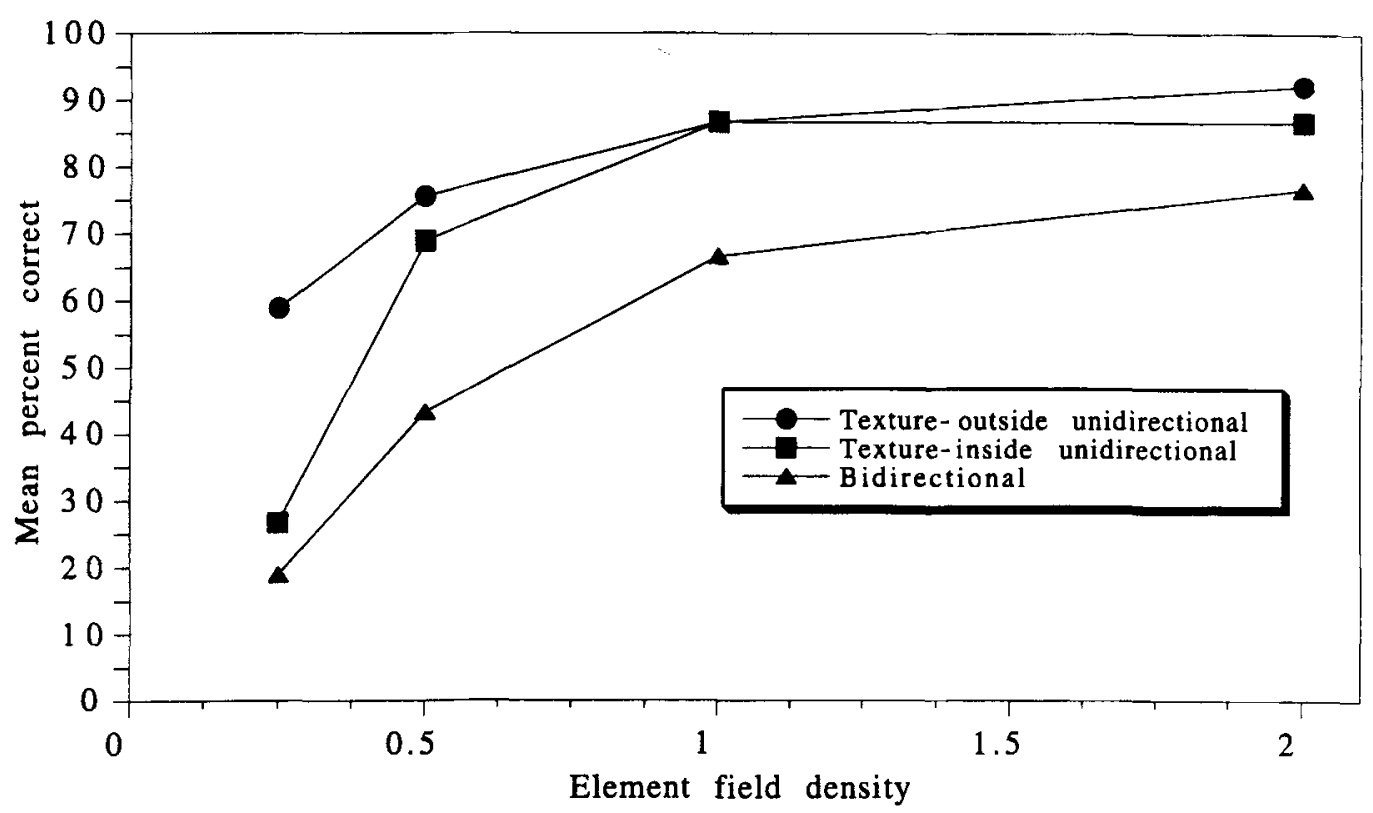

Figure 3. Shape identification accuracy plotted as a function of element density for the three conditions in Experiment 1. 
tween texture-inside and texture-outside displays disappeared at higher densities.

The results of Experiment 1 raise two questions. First, why was boundary formation impaired in the textureinside displays but not in the texture-outside displays? Second, why did this impairment occur only at low densities? A complete answer to the first question may require an answer to the second question. The simplest explanation for why a difference between texture-inside and textureoutside displays is seen only at low densities would be that the equivalence of texture-inside and texture-outside displays at high densities reflects a ceiling effect. As accuracy rises with increasing texture density, the textureoutside accuracy simply hit the performance ceiling before the texture-inside accuracy. Although a ceiling effect may partially explain the similarity of performance at high densities, it is unlikely to be the entire explanation. A ceiling effect does not explain, for example, why the shapes of the density curves for the bidirectional and texture-outside displays were so similar, whereas both differed dramatically from the texture-inside curve (Figure 3 ). This divergence suggests that something occurred in the texture-inside displays as density increased that did not happen in the other two types of displays.

It is possible that as density increases, there is a change in the figure-ground relations in the texture-inside displays. Specifically, at low densities the dynamically defined contours may be seen to bind inward so that the static internal texture will be seen as being on the surface of the moving form. In contrast, at higher densities the contours bind outward toward the empty black region; here, the internal texture would appear to be visible through an aperture. If conflict between the motion of texture and a dynamically defined edge affects SBF only when the texture is seen as a part of the surface bounded by the moving edge, then the perceptual assignment of contour direction (and thus the determination of figure and ground) will be directly related to whether or not motion conflicts interfere with boundary formation in these displays. Thus, a change in figure-ground segmentation of the texture-inside displays from a moving form with static texture on its surface to a large occluder with a hole in it would remove the motion conflict.

The specific role of boundary assignment, and, more generally, figure-ground segregation, has received relatively little attention in research on dynamic boundary formation. Kaplan's (1969) work sheds some light on the role of texture change in assigning contour direction. He noted that when elements appear or disappear on only one side of an edge, the contour appears to bind to the unchanging surface, and that surface is seen as closer than the other. When elements appear or disappear on both sides of an edge, the contour does not appear to belong to either surface. Kaplan summarized his subjects' descriptions of the latter type of display as "looking as if there were two textured surfaces going around rollers that abutted at a crack" (Kaplan, 1969, p. 196). Thus, stationary contours were seen as belonging to the adjoining sta- tionary texture field, and when both fields moved, the contour did not bind to either field.

More recently, Yonas and colleagues (Craton \& Yonas, 1988, 1990; Yonas, Craton, \& Thompson, 1987) suggested that the relative motion between a surface and an edge (they refer to this type of relative motion as boundary flow) can be used to determine whether that surface and edge are seen as grouped together. Specifically, a contour will bind to texture whose motion is identical to the motion of the contour, and away from texture with a dissimilar motion. The assignment of contour direction from boundary flow occurs with both luminance-defined edges and spatially defined illusory contours (Yonas et al., 1987). Although Kaplan's (1969) and Yonas's theories offer somewhat different accounts for which aspects of dynamic displays are critical for determining the direction of contour binding, they make substantially different predictions only when texture and edges are not close (Craton \& Yonas, 1990). For our purposes, they make identical predictions about contour assignment in the SBF displays employed in these experiments. So, we use the term boundary flow to refer to them both.

A boundary flow analysis of Experiment l's displays would predict that the contours seen in texture-inside displays should bind outward, away from the static texture, which does not move with the dynamically defined edge. In the texture-outside displays, the motion of the dynamically defined edge relative to the static texture should result in a contour that binds inward. In the bidirectional displays, where the dynamically defined edge changes location relative to both the interior and exterior texture, the contour may not bind consistently to either surface. Such displays, where contour assignment would be uncertain and might fluctuate, should be weaker than displays where boundary assignment is stable over time. Such fluctuations might also account for the tendency to identify all forms (even the smooth ones) as irregular. Thus, boundary flow would appear to be able to account for the equivalent clarity of texture-inside and textureoutside displays at high densities, as well as the consistently lower clarity in the bidirectional display, where stable edge assignments would not be possible.

An account based solely on boundary flow, however, would suggest that the relative contour stability should remain constant as element field density decreased, since the motion patterns in the three types of display do not change as a function of density. Yet, this is not what happens. Perhaps aspects of the texture-inside displays other than relative motion lead the contours to bind inward at low densities. In particular, the Gestalt principles of figure-ground relations would suggest that the contours in these displays should bind inward because contours tend to bind in the direction of the smallest enclosed area $(\mathrm{Ru}-$ bin, 1915/1958).

A change in figure-ground organization in dynamic displays may occur as density changes because the effect of boundary flow on boundary assignment may increase proportionally as texture density increases and, at some 
point, becomes more important than the other, static, sources of figure-ground information. That is, increases in contour strength may increase the effectiveness of boundary flow. Since low density displays do not define the edges very well (the relatively poor form identification accuracies at low densities, for all three types of displays in Experiment 1, suggest that edges were indeed not well defined), the contours seen at lower densities might be insufficient to allow boundary flow information to determine binding. Although there has not been any systematic research on the effect of contour clarity on boundary flow, strong contours appear to be necessary for other unit formation phenomena where boundaries and texture are grouped. Ramachandran (1985) and Ramachandran and Anstis (1986) have found that texture capture in apparent motion occurs only with well-defined boundaries (either luminance-defined or static illusory boundaries); weakly defined boundaries do not bind with the enclosed surface. Thus, in texture-outside displays and low-density texture-inside displays, the contours will bind inward, in accordance with Gestalt principles. For the texture-outside displays, this presents no problems, but in the textureinside displays this results in a motion conflict between the nascent SBF edges and the texture seen as being on the surface bound to those edges. As the spatial density of transformations increases and the contours increase in

(a)

Texture-outside unidirectional

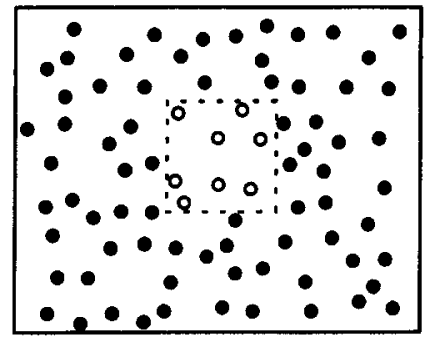

(c)

Texture-inside annulus unidirectional

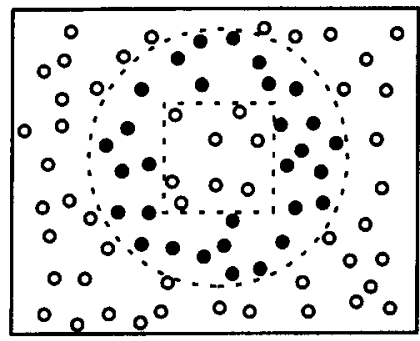

- =Visible texture element clarity, the boundary flow information may cause the contours in the texture-inside displays to bind outward, effectively removing the motion conflict. We explore the interaction between boundary assignment and edge perception in Experiments 2 and 3.

\section{EXPERIMENT 2}

If a change in the figure-ground organization was responsible for the change in the clarity of the spatiotemporally defined form in the texture-inside displays, then any information that changes the direction of contour binding in these types of displays should affect contour clarity. In Experiment 2, the direction of binding was manipulated by adding a second dynamically defined edge that surrounded the forms used in Experiment 1. This produced two new types of display: Texture-inside-annulus unidirectional and texture-outside-annulus unidirectional displays. Texture-outside-annulus displays were generated by adding an additional edge to the texture-inside displays (Figures $4 \mathrm{~b}$ and $4 \mathrm{~d}$ ). In these displays, the elements inside the inner edge (the edge to be identified) were visible, as were the elements outside the outer edge. Note that both the texture-outside-annulus and the textureinside displays had visible elements enclosed by the edge to be identified. In a texture-outside-annulus display, the

(b)

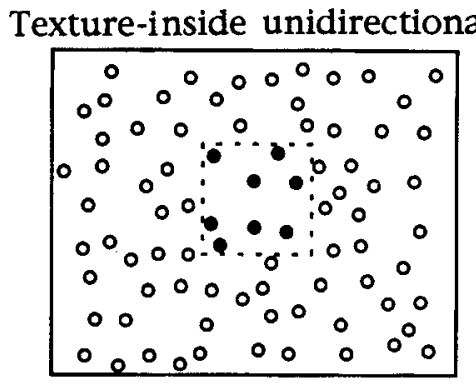

(d)

Texture-outside annulus unidirectional

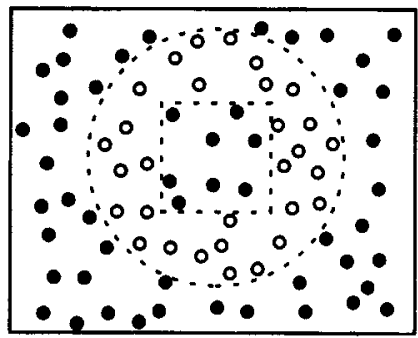

$0=$ Invisible texture element

Figure 4. Illustration of the four conditions used in Experiment 2. The (a) texture-outside and (b) texture-inside conditions were identical to those used in Experiment 1. For the (c) texture-inside-annulus and (d) texture-outsideannulus conditions, a dynamically defined circular edge enclosed the pseudosurface. 
outer contour would be attached to the smallest enclosed area if it bound inward. Since the common motion of the two edges should help to group them, the inner contour should bind toward the outer edge. Thus, the textureoutside-annulus displays should reduce the motion conflict that is present in the low-density texture-inside displays, and the clarity and stability of the inner contour should increase.

Likewise, texture-inside-annulus displays were generated by adding the additional outer edge to texture-outside displays (see Figures $4 \mathrm{a}$ and $4 \mathrm{c}$ ). Both the texture-insideannulus and the texture-outside displays had no visible elements inside the edge to be identified. Just as the addition of an outer edge to the texture-inside displays should aid in binding the inner contour outward and thus remove the motion conflict, so the addition of a second edge to texture-outside displays might cause the inner contour to bind toward the outer edge. The static texture between the two edges would then be in conflict with the motion of the edges. This should decrease the clarity and stability of the inner contours in the texture-inside-annulus displays.

\section{Method}

Subjects. Thirteen Temple University undergraduates participated in partial fulfillment of introductory psychology class requirements. Two subjects' data were excluded from analysis due to the subjects' failure to follow instructions.

Apparatus. The apparatus was identical to that used in Experiment 1, except that a Macintosh Quadra $840 \mathrm{AV}$ replaced the Macintosh Quadra 800.

Displays. The displays were identical to those used in Experiment 1 with the following three exceptions. First, only the $0.25 \%$, $0.5 \%$, and $1 \%$ density levels were used. Second, the size of the display area was doubled. This was necessary to ensure that the outer edge was always on the screen. Third, the bidirectional condition was replaced with two new conditions: the texture-inside-annulus and texture-outside-annulus conditions. The texture-outside-annulus condition was similar to the texture-inside condition: Elements appeared at the leading edge and disappeared at the trailing edge of the form to be identified - the pseudosurface. Unlike the texture-inside displays, the texture-outside-annulus displays had a circular edge surrounding the pseudosurface. The circular edge was $5.71 \mathrm{~cm} \mathrm{(2.18} \mathrm{arc}$ deg) from the center of the pseudosurface. Elements disappeared at its leading edge and appeared at its trailing edge. As a consequence, elements in these displays were visible only when they were outside this new edge or inside the pseudosurface.

Likewise, the texture-inside-annulus condition was identical to the texture-outside condition except that a circular edge was added that surrounded the pseudosurface. So, for both the texture-outside and texture-inside-annulus conditions, elements disappeared at the leading edge and appeared at the trailing edge of the pseudosurface.

Crossing four display types (texture outside, texture inside annulus, texture inside, and texture outside annulus), three element field densities $(0.25 \%, 0.5 \%$, and $1 \%)$ and 10 pseudosurfaces yielded 120 trials. The 120 trials were presented in random order.

Procedure. The procedure was identical to that used in Experiment 1 , with the following exception. Subjects were informed that occasionally more than one boundary would be present. The experimenter orally emphasized the fact that the subject was to identify the innermost edge, if more than one edge was present.

\section{Results}

The addition of a second edge, which we hypothesized would reverse the boundary assignment for the contour to be identified, had the predicted effects on accuracy in the two unidirectional displays employed in Experiment 1. Adding a second boundary to the texture-inside displays increased accuracy rates from $61 \%$ to $71 \%$ at the lowest density. Adding a second boundary to the texture-outside displays decreased accuracy at this density from $77 \%$ to $68 \%$.

Mean accuracies for the four types of displays are plotted as a function of element field density in Figure 5. A three-way ANOVA was performed on accuracy rates, with the location of texture elements (inside or outside) relative to the edge to be identified, presence versus absence of an additional edge, and element field density as within-subject factors. A significant main effect was found for element location $[F(1,10)=6.34, p<.03]$ : Overall performance for displays that had texture inside the pseudosurface (the texture-inside and the textureoutside-annulus displays) was lower than for displays with texture outside the pseudosurface (texture-outside and texture-inside-annulus displays). A main effect was also found for element field density $[F(2,20)=15.29, p<$ $.0001]$ : Accuracy increased with increases in density. The main effect of an additional edge was not significant $[F(1,10)=2.08, p>.15]$. This is noteworthy because it means that the addition of the extra edge did not bias subjects' responses: The presence of a large, dynamically defined circle surrounding the form to be identified did not increase the number of times subjects mistakenly identified the inner edge as a circle.

The only interaction that was significant was the additional boundary $\times$ element location interaction $[F(1,10)=$ $13.44, p<.0001$; all other $F \mathrm{~s}<1.60, p \mathrm{~s}>.20]$. This interaction was explored with planned comparisons. Overall, texture-outside-annulus accuracy was significantly higher

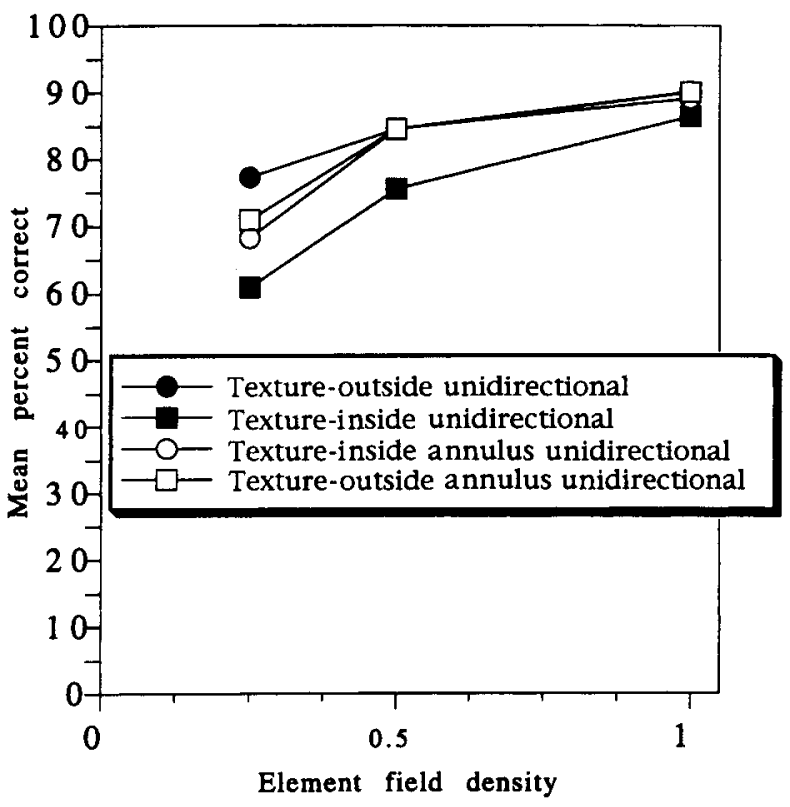

Figure 5. Shape identification accuracy plotted as a function of element density for the four conditions in Experiment 2. 
than texture-inside accuracy $[F(1,10)=12.27, p<.006]$. Adding information that could remove conflicting motion information by altering the direction of contour binding improved shape identification performance, with larger improvements at lower densities. Although the overall difference between texture-inside-annulus and textureoutside accuracy did not reach significance $[F(1,10)=$ $2.829, p<.15]$, these displays exhibited the same pattern as that found in the texture-inside and texture-outside displays: Larger differences were found at low densities, and small or no differences were found at higher densities. Performance on the texture-inside-annulus displays was significantly below performance on the texture-outside displays at the $0.25 \%$ density level $[F(1,20)=5.21, p<$ $.04]$, but was not significantly different at the higher two densities (both $F \mathrm{~s}<1$ ).

\section{Discussion}

Motion conflict between a moving figure and the stationary texture that is seen as a part of that figure influences contour clarity. The addition of a second dynamically defined edge that moved with the figure to the texture-inside displays (making them texture-outside-annulus displays) aided in binding the inner contour away from the stationary elements. As predicted, this increased the clarity of the spatiotemporal contour and eliminated the instability seen in the texture-inside displays. Likewise, the addition of a second edge to the texture-outside displays, making them textureinside-annulus displays, reduced contour clarity. Notably, the addition of a second edge had the largest effect at low densities. This parallels the findings of Experiment 1, where texture-inside and texture-outside displays differed only at low densities. At high densities, boundary flow information in the texture-inside-annulus displays and the texture-inside displays may specify the direction of binding and remove the motion conflict.

Thus, SBF is considerably impaired when a conflict is introduced between the motion of a figure's dynamically defined edge and its surface texture. If the lack of motion of elements seen on a figure's surface impairs boundary formation, then boundary formation might be facilitated by the addition of elements that move with the edge. Experiment 3 tested this hypothesis and further investigated the role of boundary assignment in SBF.

\section{EXPERIMENT 3}

In Experiment 3, consistent motion information was added to the texture-outside and the texture-inside displays in the form of eight small elements that had the same motion as the figure. The same spatial pattern of eight elements was used for all displays, so the elements provided information about the motion but not the shape of the moving form. If motion signals that are spatially distant from the edges can be used in SBF, then the additional moving elements should counter the effects of the stationary texture in the texture-inside displays. As a result, con- tours should appear clearer than those seen in the textureinside displays without moving elements. To the extent that additional motion information helps stabilize boundary formation it should also facilitate boundary formation, in the texture-outside displays.

In addition to investigating the effect of additional consistent motion, the location of the motion was manipulated. Experiments 1 and 2 provided some evidence that the visual system may restrict which motion signals influence boundary formation. In any display with moving edges and stationary texture, there is the potential for a motion conflict between the texture and the edges. It appears that the direction of binding determines whether or not such a conflict will arise: Only when the moving contour is seen as belonging to the region with the stationary texture will the motion (or lack thereof) of the texture have an effect. To test this spatial-restriction-by-boundaryassignment hypothesis, two groups of subjects were run in Experiment 3. For the first group (inside motion), the eight moving elements were inside the form to be recognized (Figure 6a). In the second group (outside-motion), the eight elements were outside the form (see Figure 6b). If the effect of element motion on boundary formation is restricted, the two groups should differ in how additional moving elements affect texture-inside and texture-outside displays. Since the contours in the texture-inside displays bind inward under some conditions and bind outward under other conditions, a benefit of extra motion may be seen in both the inside-motion and outside-motion groups. The contours in the texture-outside displays have only been observed to bind inward, so only the inside-motion group should benefit from the extra motion. It is possible that the motion of the elements might influence the direction of boundary assignment in these displays, on the basis of boundary flow, so that the contour would bind toward elements that share its motion. However, the moving elements would be expected to have little influence relative to the effects of the static elements since, even in the lowest density displays, the number of static elements is much greater than the number of moving elements.

\section{Method}

Subjects. Twenty Temple University undergraduates participated in partial fulfillment of introductory psychology class requirements.

Apparatus. The apparatus was identical to that used in Experiment 2.

Displays. The displays were identical to those used in Experiment 1 , with the following two exceptions. First, the bidirectional condition was not included. Second, half of the displays contained extra motion information in the form of eight elements, which translated along with the pseudosurface.

For half of the subjects (inside motion), the extra elements were inside the pseudosurfaces (Figure 6a). In order to use the same pattern of elements for all figures, a pattern was selected so that the eight elements were inside all 10 pseudosurfaces. The pattern was further constrained by selecting locations so that the set of elements, as a group, did not resemble any of the pseudosurfaces.

For the other half of the subjects (outside motion), the eight additional elements were outside of the figure. The same relative element positions used for the inside-motion displays were employed, 
Texture-outside unidirectional

(a)

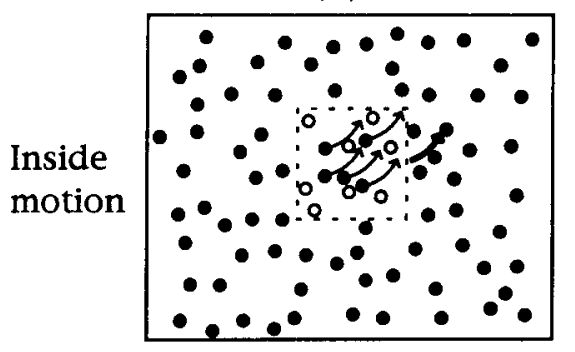

(c)

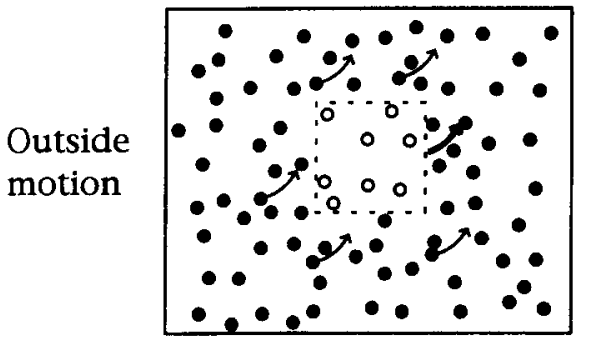

- =Visible texture element

$\checkmark=$ Moving texture element
Texture-inside unidirectional

(b)

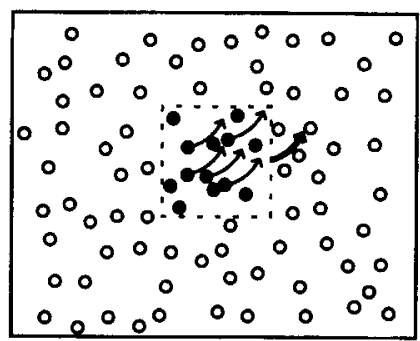

(d)

$\left.\begin{array}{|llllllllll}\hline & 0 & 0 & 0 & 0 & 0 & 0 & & 0 \\ 0 & 0 & 0 & 0 & 0 & 0 & 0 & 0 & 0 \\ 0 & 0 & 0 & 0 & 0 & 0 & 0 & & \\ 0 & 0 & 0 & 0 & 0 & 0 & 0 & 0 \\ 0 & 0 & 0 & 0 & 0 & 0 & 0 & 0 & 0 \\ 0 & 0 & 0 & 0 & 0 & 0 & 0 & 0 & 0 & 0 \\ 0 & 0 & & 0 & 0 & 0 & 0 & 0 & 0 \\ 0 & 0 & 0 & 0 & 0 & 0 & 0 & 0 & 0 & 0\end{array}\right]$

$0=$ Invisible texture element

soving pseudosurface

Figure 6. Illustration of the extra elements in Experiment 3. Eight elements moved with the pseudosurface in texture-outside ( $a$ and $c$ ) and texture-inside ( $b$ and d) displays. Moving elements were positioned inside the pseudosurface for half of the displays ( $a$ and $b$ ) and outside in the remaining displays ( $c$ and $d$ ).

but the distance between elements was increased so that the elements were outside all 10 pseudosurfaces (Figure 6b).

Combining two types of transformation (texture outside and texture inside), presence and absence of additional moving elements, 10 figures, and four densities yielded 160 trials. These were presented in random order.

Procedure. The procedure was identical to that used in Experiment 1 , with the following exception. Both the written and verbal instructions mentioned that several extra dots might move around the screen. The experimenter orally instructed the subjects to ignore the extra dots and to report the shape of the moving form.

\section{Results}

Shape identification accuracies for the four groups are plotted as a function of density in Figure 7. Adding consistent motion information increased shape identification accuracy. When the extra motion information was present inside the figure (Figure 7a), overall accuracy increased, from $77 \%$ to $82 \%$ for the texture-outside displays, and from $61 \%$ to $67 \%$ for the texture-inside displays. When the information was present outside the figure (Figure $7 \mathrm{~b}$ ), only texture-inside accuracy increased $(57 \%-67 \%)$.

The mean accuracies were subjected to a four-way ANOVA with transformation type (texture outside and texture inside), presence or absence of moving elements, and element field density as within-subject factors, and motion location (inside or outside motion) as a between- subjects factor. As was found in Experiments 1 and 2, the main effects for density and transformation were significant $[F(2,36)=253.14, p<.0001$, and $F(1,18)=66.23$, $p<.0001$, respectively]. Identification was easier at higher densities and the forms were easier to identify in the texture-outside displays than they were in the texture-inside displays. As was also found in Experiments 1 and 2, the transformation $\times$ density interaction was significant $[F(2,36)=17.04, p<.0001]$ : The difference between performance in the texture-inside and the texture-outside displays disappeared at higher densities.

The main effect for additional motion was significant $[F(1,18)=8.05, p<.02]$ : Adding motion information that was consistent with the motion of the edges increased shape identification accuracy. Although the main effect for motion location was not significant $(F<1)$, the threeway interaction among location, transformation, and additional motion was significant $[F(1,18)=4.77, p<.05]$. This interaction is consistent with the spatial-restrictionby-boundary-assignment hypothesis: The addition of moving elements increased accuracies for texture-inside displays regardless of whether the moving elements appeared inside or outside the pseudosurface, but facilitated shape perception in texture-outside displays only when they were inside (Figure 7). There was a small decrease in performance when the motion information was outside 
(a)

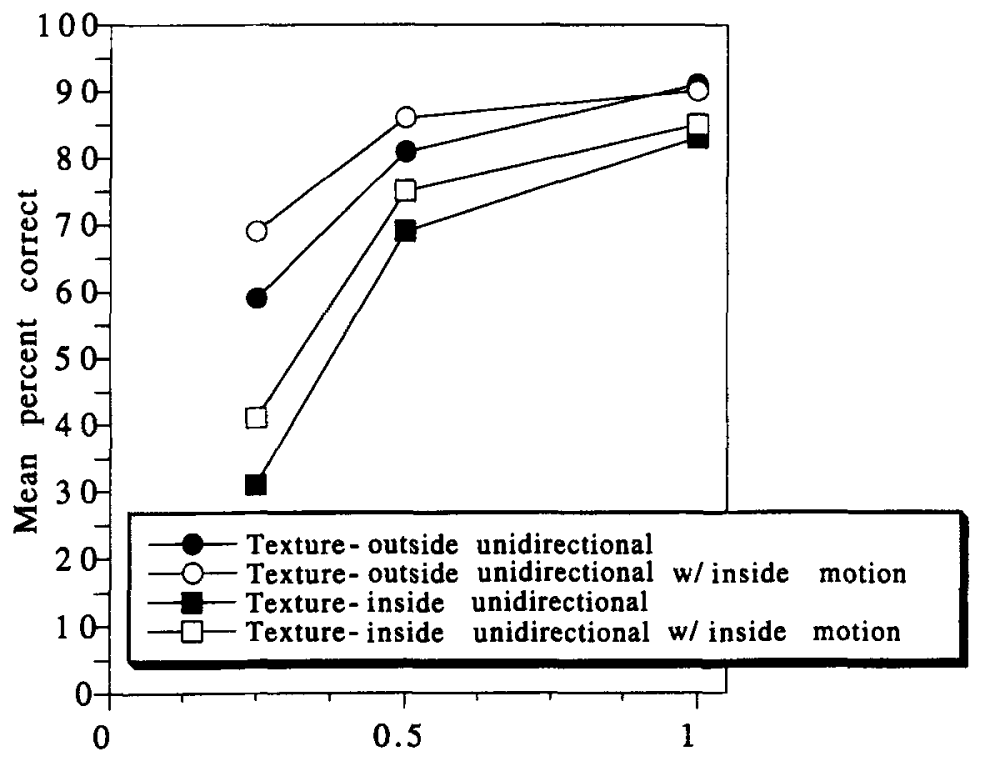

(b)

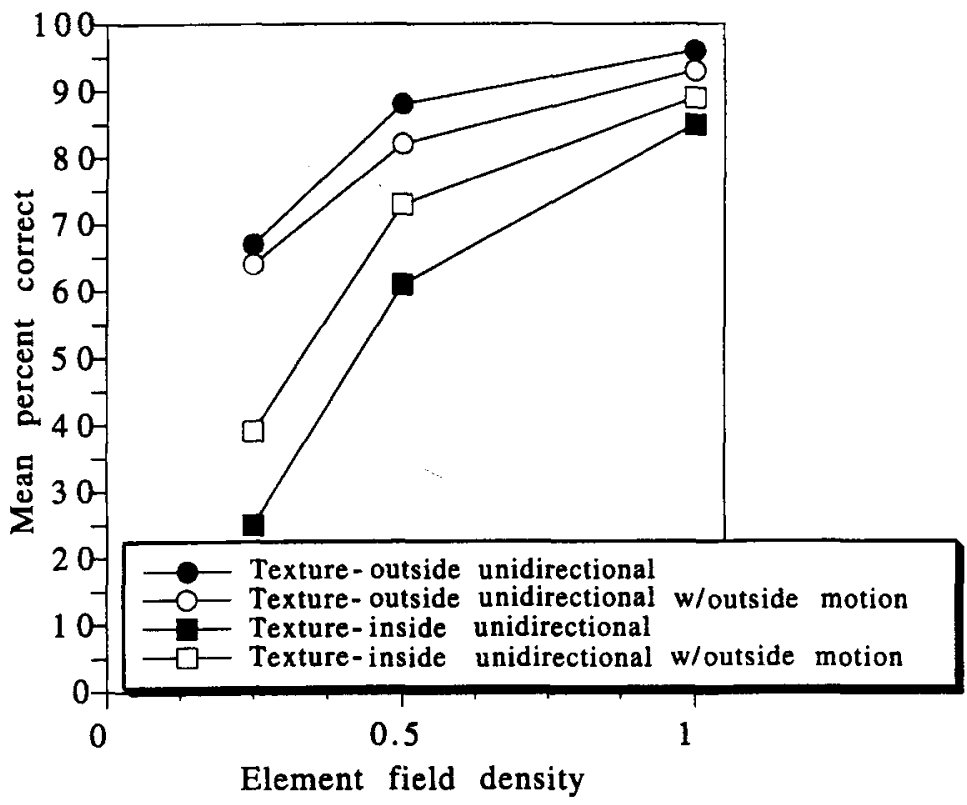

Figure 7. Shape identification accuracy for Experiment 3 plotted as a function of element density for (a) the inside-motion group and (b) the outside-motion group.

the pseudosurface in texture-outside displays that did not reach significance [from $84 \%$ to $80 \%, F(1,9)=1.18$, n.s.].

\section{Discussion}

The spatial-restriction-by-boundary-assignment hypothesis was supported by the results of Experiment 3 . The addition of a consistent motion signal increased contour stability and clarity, but only when the additional moving elements were grouped with the figure's edges. The addition of a consistent motion signal to the texture- outside displays, where the contours bind inward, aided shape perception only when the moving elements were enclosed by the figure, and may have interfered with shape perception when they were outside. In texture-inside displays, placing consistent global motion information inside the figure increased performance just as much as adding it outside. This is consistent with the dual nature of texture-inside displays: The contours in a texture-inside display can bind inward or outward. Additional motion facilitates shape perception in either case. 
(a)

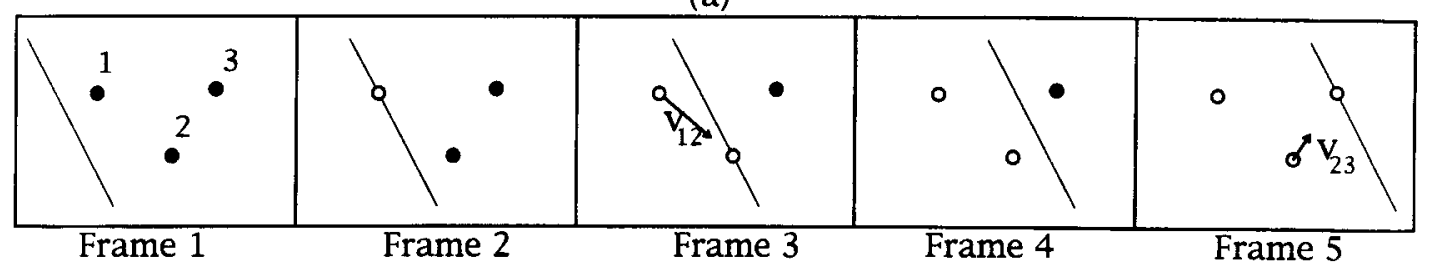

(b)

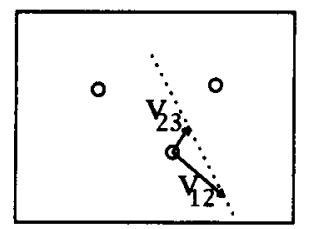

Figure 8. (a) An illustration of an edge sequentially occluding three texture elements. Each pair of disappearances defines a motion vector, which is illustrated with arrows. The two motion vectors, v12 and v23, can be combined, as shown in (b), to define the orientation of the occluding edge. From "Spatiotemporal Boundary Formation: The Role of Local Motion Signals in Boundary Perception," by T. F. Shipley and P. J. Kellman, 1997, Vision Research, 37 , p. 1287. Copyright 1997 by Elsevier Science. Reprinted with permission.

\section{GENERAL DISCUSSION}

The three experiments reported here demonstrate that the motion (or lack thereof) of texture elements directly affects the perceptual clarity and stability of dynamically defined contours. They also show that only the motion of elements seen as part of a figure influences the formation of the boundaries of that figure; the motion of elements that are seen as part of other figures has little or no effect on dynamic boundary formation. Thus, it seems that the clarity of the shape of an object is determined, in part, by figure-ground organization. Treating a hole as if it were a surface and binding the stationary internal texture to its moving edge will interfere with recovering the shape of the hole.

Current models of SBF (e.g., Bruno \& Gerbino, 1991, Shipley \& Kellman, 1997) focus on the role of the local motion signals that arise at the edges of moving surfaces; however, it is possible to integrate information about the global motion of the form into these models. For example, Shipley and Kellman $(1994,1997)$ suggested that the contours seen in SBF displays are best accounted for by a motion-before-form model in which local motion signals, defined by pairs of abrupt element transformations, serve as the basis for local boundary formation. Figure 8 illustrates the idea that transformation-based local motion signals can represent both the spatial and temporal separation between two element transformations (e.g., appearances or disappearances) along a moving edge, and that the pattern of such motion signals defines the local orientation of the moving boundary. Specifically, if the two vectors representing the local motion signals between three sequential element changes are arranged so that they have a common origin, the line defined by the tips of the vectors has the same orientation as the moving edge. Shipley and Kellman (1997) offered a proof that occlusion of as few as three noncollinear elements can define the orientation of an edge whose velocity is not known (Figure 8). When the edge's velocity is known, a corollary of this proof based on substituting its velocity (both its direction and speed of motion) for one of the local motion signals demonstrates that two elements are sufficient to define the edge's orientation. Thus, surface texture motion (e.g., the moving elements used in Experiment 3 ) could facilitate the extraction of local boundary segments by providing information about the direction and speed of motion of the local segments. This would explain why only texture elements seen as a part of the figure affect the perception of that figure's contours.

According to this account, the effects of consistent texture motion on boundary formation should be particularly evident in low-density displays, where the spatiotemporal density of element changes is near threshold levels. In these cases, the addition of edge velocity information may make the difference between a contour being seen or not. Consistent element motion may also increase performance slightly at higher density levels by increasing the number of motion signals available for local boundary orientation extraction. The same analysis would apply for inconsistent motion and thus would account for why the effects seen in these experiments were most likely to occur at low densities, at which surface motion information would be most influential.

This account does not identify which texture elements will be seen as belonging to which contours and hence when motion signals will be grouped with that contour. Which local boundary segments and texture elements belong together may be determined by static properties, such as enclosure, size, and symmetry (Rubin, 1915/1958), as well as dynamic properties, such as where changes occur 
and common fate (Craton \& Yonas, 1990; Kaplan, 1969; Yonas et al., 1987). Once elements are grouped with a boundary segment, the motion of those elements may contribute to the perceived motion of the entire group. Information about the motion of a boundary segment should aid in extracting the orientation of that boundary segment, so appropriate grouping should facilitate SBF and inappropriate grouping should interfere with SBF.

Thus, differences in direction of binding may account for, at least in part, the phenomenal and performance differences between bidirectional and texture-outside displays. Yet, the presence of static texture both inside and outside of a figure is not sufficient to impair boundary formation. In one of the conditions reported by Shipley and Kellman (1994), a set of texture elements on a black background changed from white to blue. Even though all the static elements were always visible in these color transformation displays, the boundaries seen in the unidirectional displays (where blue texture elements were visible inside) differed in clarity from the bidirectional displays (where the texture inside and outside was a mix of blue and white elements). Furthermore, the boundaries seen in the unidirectional displays where the elements were white outside and blue inside did not differ in clarity from texture-outside displays, where elements disappeared entirely inside the moving form (Shipley \& Kellman, 1994).

Although it is possible that the static blue elements had little effect because their luminance was very low, an alternative account is suggested by the phenomenal appearance of these color transformation displays. In the unidirectional displays where the elements were white outside and blue inside, the moving form appeared to be a blue film, with static, white elements seen through the film. Such a percept suggests that there may be some information in these dynamic displays that can specify a moving, partially opaque surface located in front of a field of static texture elements (Cicerone et al., 1995; Cunningham et al., in press). It has recently been shown that changes over time can define the opacity of surfaces (Cunningham et al., in press; Stoner \& Albright, 1996). Stoner and Albright suggested that contrast modulation was a source of information for surface opacity, and Cunningham et al. suggested that local changes in color of texture elements might also be used to identify the color of a moving, partially opaque surface.

The dynamic specification of surface quality suggests an alternative to the direction-of-binding explanation for why performance in the texture-inside displays was good at high densities. Here, instead of seeing a moving hole, several subjects reported that the forms had the appearance of moving flashlight beams. This suggests that SBF can also define the boundaries of regions of varying brightness (see Fuchs (1923/1950) for a discussion of perception of transparency and shadows in static displays), and the spatiotemporal pattern of changes (in this case, local increases in luminance) defines the brightness of the region. The appearance of a bounded region of higher brightness, which occurred in high-density texture-inside displays, would remove the effect of static texture because the texture is no longer seen as part of the moving form. We are currently investigating the conditions under which dynamic information may define partially opaque surfaces, as well as regions that appear luminous.

One of the central problems of static object perception is offering an account for how all aspects of an object are unified into a signal coherent perceptual whole (see, e.g., Treisman \& Gelade, 1980). Moving objects offer a formally similar problem with the additional complexity introduced by the potential for aspects of the object to be revealed at different points in time. Integration under such circumstances would require combining information over space and time. We have suggested here that boundary formation processes may take advantage of transformationbased, locally defined motion information as well as spatially remote surface motion information. This may allow integration to occur over large spatial scales. Furthermore, local motion signals and surface motion information may be used to determine other aspects of the moving object, such as surface quality (Cunningham et al., in press; Stoner \& Albright, 1996). Perhaps one of the ways the binding problem is solved for moving objects is by using motion signals as the "glue" for various aspects of the unit.

\section{REFERENCES}

Andersen, G. J., \& Cortese, J. M. (1989). 2-D contour perception resulting from kinetic occlusion. Perception \& Psychophysics, 46, 4955.

Bruno, N., \& Bertamini, M. (1990). Identifying contours from occlusion events. Perception \& Psychophysics, 48, 331-342.

Bruno, N., \& GERBINo, W. (1991). Illusory figures based on local kinematics. Perception, 20, 259-274.

Cicerone, C. M., Hoffman, D. D., Gowdy, P. D., \& Kim, J. S. (1995) The perception of color from motion. Perception \& Psychophysics, 57, $761-777$.

Craton, L. G., \& Yonas, A. (1988). Infant's sensitivity to boundary flow information for depth at an edge. Child Development, 59, 1522-1529.

Craton, L. G., \& Yonas, A. (1990). Kinetic Occlusion: Further studies of the boundary-flow cue. Perception \& Psychophysics, 47, 169-179.

Cunningham, D. W., Shipley, T. F., \& Kell.man, P. J. (in press). The dynamic specification of surfaces and boundaries. Perception.

Fuchs, W. (1950). On transparency. In W. D. Ellis (Ed.), A source book of Gestalt psychology (pp. 89-94). New York: The Humanities Press. (Original work published 1923)

Gibson, J. J. (ProduCER) (1968). The change from visible to invisible. A study of optical transitions [Film]. (Available from Psychological Cinema Register, State College, PA)

Gibson, J. J., Kaplan, G. A., Reynolds, H. N., Jr., \& Whefi.er, K. (1969). The change from visible to invisible: A study of optical transitions. Perception \& Psychophysics, 5, $113-116$.

HelmHOLJZ, H. von (1962). Handbook of physiological optics (3rd ed., J. P. C. Southal!, Trans.) New York: Dover. (Original work published 1867)

HiNE, T. (1987). Subjective contours produced purely by dynamic occlusion of sparse-points array. Bulletin of the Psychonomic Society, 25, 182-184.

KaPlaN, G. A. (1969). Kinetic disruption of optical texture: The perception of depth at an edge. Perception \& Psychophysics, 6, 193-198.

Michotte, A., Thines, G., \& CrabBÉ, G. (1991). The amodal completion of perceptual structures. In G. Thines, A. Costall, \& G. Butterworth (Eds.), Michotte 's experimental phenomenology of perception 
(pp. 140-168). Hillsdale, NJ: Erlbaum. (Original work published in 1964)

Miyahara, E., \& Cicerone, C. M. (1997). Chromaticity and luminance contribute to the perception of color from motion. Perception, 26, 1381-1396.

Ono, H., Rogers, B. J., Ohmi, M., \& Ono, M. (1988). Dynamic occlusion and motion parallax in depth perception. Perception, 17, 255-266.

RAMACHANDRAN, V. S. (1985). Apparent motion of subjective surfaces. Perception, 14, 127-134

RAMACHANDRAN, V. S., \& ANSTIS, S. (1986). Figure-ground segregation modulates apparent motion. Vision Research, 26, 1969-1975.

RogERS, B. J. (1984). Dynamic occlusion, motion parallax and the perception of 3-D surfaces. Perception, 13, A46.

Rogers, B. J., \& Graham, M. E. (1983). Dynamic occlusion in the perception of depth structure. Perception, 12, A15.

RoYden, C. S., Baker, J. F., \& Allman, J. (1988). Perceptions of depth elicited by occluded and shearing motions of random dots. Perception, 17, 289-296.

RuBin, E. (1958). Figure and ground. In D. C. Beardslee \& M. Wertheimer (Eds.), Readings in perception (pp. 194-203). Princeton, NJ: Van Nostrand. (An abridged translation by M. Wertheimer of Visuell wahrgenommene Figuren, 1921, Copenhagen: Gyldendal, which was a translation from Danish to German by P. Collett of Synsoplevede figurer, 1915, Copenhagen: Gyldendal)
Shipley, T, F, \& Kellman, P. J. (1993). Optical tearing in spatiotemporal boundary formation: When do local element motions produce boundaries, form, and global motion? Spatial Vision, 7, 323-339.

Shipley, T. F., \& Kellman, P. J. (1994). Spatiotemporal boundary formation: Boundary, form, and motion perception from transformations of surface elements. Journal of Experimental Psychology: General, $123,3-20$.

ShiPLEy, T. F., \& Kellman, P. J. (1997). Spatiotemporal boundary formation: The role of local motion signals in boundary perception. $\mathrm{Vi}$ sion Research, 37, 1281-1293.

STAPPERS, P. J. (1989). Forms can be recognized from dynamic occlusion alone. Perceptual \& Motor Skills, 68, 243-251.

StONER, G. R., \& AlBright, T. D. (1996). The interpretation of visual motion: Evidence for surface segmentation mechanisms. Vision Research, 36, 1291-1310.

Treisman, A., \& Gelade, G. (1980). A feature-integration theory of attention. Cognitive Psychology, 12, 97-136.

Yonas, A., Craton, L. G., \& Thompson, W. B. (1987). Relative motion: Kinetic information for the order of depth at an edge. Perception \& Psychophysics, 41, 53-59.

(Manuscript received December 17, 1996; revision accepted for publication July 5,1997 .) 